\title{
Using Technologies to Support Reminiscence
}

\author{
Dan Cosley ${ }^{1}$, Kathy Akey ${ }^{2}$, Brian Alson ${ }^{1}$, Jonathan Baxter ${ }^{1}$, \\ Mark Broomfield ${ }^{1}$, Soyoung Lee ${ }^{1}$, Chethan Sarabu ${ }^{1}$ \\ ${ }^{1}$ Cornell University Information Science; ${ }^{2}$ New York University \\ \{drc44, bda9, jtb42, mbb89, sl426, crs49\}@ cornell.edu; kda233@ nyu.edu
}

\begin{abstract}
This paper is about the evolution of a system prototype called Pensieve whose goal is to support people's reminiscing practices. A number of technologies exist to manage memory-related content; however, these technologies tend to take a model of memory as information that leads to a focus on capture and access. Pensieve is instead based on reusing memory-laden content people already create in social media services. This idea is supported by theories of autobiographical memory, insights from interviews with eight subjects, and experiences with two prototypes deployed to ten users. These interviews and experiences suggest that people value even simple tools that support reminiscence, as well as providing both design goals and research questions around the design of tools that support people in reminiscing.
\end{abstract}

\section{Categories and Subject Descriptors}

H.5.2 [nformation Interfaces and Presentation (e.g., HCI)]: User Interfaces

\section{Keywords}

reminiscence, memory, social media, personalization

\section{INTRODUCTION}

The other day, someone commented on a Facebook photo one of the authors had been tagged in. This led him to look at the photo and reflect on when it was taken, the people who were in it, and the activities he was engaged in at the time of the picture; in short, to reminisce about the past. This was pleasant, and in fact, research has shown that reminiscence serves a number of positive functions throughout a person's life, including maintaining relationships, working through current situations $[2,16]$, and accepting the past. ${ }^{1}$ In therapeutic situations, reminiscing is scheduled and structured [7], but in everyday life it is generally spontaneous and

\footnotetext{
${ }^{1}$ Reminiscence can also be negative, leading to withdrawal from current activities and fueling bitter memories [15].
}

unstructured, triggered by pictures and music [17], mementos [10], nostalgic cultural icons [9], and other people.

Existing technologies that support reminiscence tend to focus on helping people manipulate and capture information. The value of photos as tools for reminiscing has led to a number of software systems (e.g., [13, 1]) or special devices (e.g., [14]) for organizing and sharing memory-laden content. Lifelogging projects such as MyLifeBits [5] attempt to capture and provide access to a person's everyday life experience through audio and video recordings, scanning documents, indexing electronic documents, and so on.

However, reminiscence is not simply manipulating information. Tools that require explicit decisions to capture or access information run contrary to the spontaneous nature of most reminiscing. Further, theories of autobiographical memory suggest the past is actively constructed and experienced differently every time it is remembered [3]. That is, it is unlikely that precise capture is needed, or perhaps even useful, for reminiscence. Some memories are bettersuited for reminiscing than others [11], and ubiquitous capture might exacerbate a problem cited by one of our informants: "I have a lot of photos that are junk."

Instead of supporting capture, we are exploring how technology can support reminiscence based on two core ideas: (1) Focus on using the memory-laden content people already create in social media systems, and (2) Explicitly remind people to reminisce using that content.

Focus on information people already capture. Rather than trying to manage the vast amounts of information lifelogging technologies can capture, systems might finesse that problem by taking advantage of people's current behavior in social media systems. When people write a blog post, publish a picture, post a message on a friend's wall, or update a status message, they are choosing to record content that is more likely than average to be important to them. For example, Sellen et al. found that photos chosen by people are much more likely to be memorable than random photos or photos automatically captured by lifelogging technologies [12]. Using the content people already create in social media systems has another important advantage in that it requires no additional effort on their part.

Remind them to reminisce by re-presenting past content. Most user-created content sites are biased toward the present: 
a user's current status, newest blog post, or most recen$\mathrm{t}$ photos. This is a natural consequence of one common use of social media, that of supporting awareness of others' current states, moods, and activities [8]. However, this focus causes previously created content to become effectively invisible. Systems might leverage this previously created content that people attend to less often, using it to remind people to reminisce (somewhat like the Ambient Ink project [6]). Re-presenting this content could naturally support the opportunistic, spontaneous nature of reminiscence.

Below, we describe the evolution of a series of prototypes based on these two ideas, the experiences of the authors, interviews with eight local community members, and the use of a prototype by ten members of our research group. These interviews and experiences led us to a number of design considerations and research questions around using technology to support reminiscence. We briefly describe those question$\mathrm{s}$, along with the current version of the prototype which we hope will help us answer them.

\section{TOOLS TO SUPPORT REMINISCENCE}

The two core ideas came from the lead author's own experience with reminiscing. He rarely remembers the past, and though he has kept a blog for the last 11 years to write down memorable or interesting observations, he rarely goes back to read it. One day, he realized that a program could remind him to read the blog entries, and further, rather than saying "read the blog", it could send specific entries to read. To maximize its spontenaiety and intrusiveness, the program randomly chose a short blog post and sent it via SMS at a random time, three to five times a day.

The interface was trivial (see Figure 1), but it effectively addressed many of the lead author's problems. It caused him to take time on a regular basis, usually just a few seconds to a minute, to reminisce. Some memories were innocent: a precocious squirrel on a trash can, which led him to think about another squirrel he had seen on a day his wife had surgery. Some were not: the first entry it ever sent was a blog entry for September 11, 2001 ("Eep"). Overall, the system led him to remember people and events that he would not have otherwise thought of, and he found this valuable.

Other people who saw the prototype and heard the idea agreed it was interesting, and pointed out a number of issues to consider. What about unpleasant memories? Could it help Alzheimer's sufferers? What about richer media? Would other people like spontenaiety, or more control? What about people who don't have digital content to reminisce about? How could we help them?

\subsection{How non-geeks reminisce}

To better understand other people's reminiscing practices, we conducted eight interviews with people outside of our research group (four male, four female, ages 25-60). We solicited participants by posting flyers throughout the community and university. The interviews lasted for about 45 minutes and participants were paid $\$ 10$. We acknowledge that our sample is both small and biased toward people who have a particular interest in either reminiscence or money. Our goal was not to draw general conclusions but to unearth design inspirations, considerations, and questions.
From: Pensieve-sender

Subject: $08 / 20 / 01$

Sue was right, I am counting the hours. Hello,

Wed. at 1:30. Goodbye, New Jersey.

Figure 1: A text message sent to the lead author from his own blog by the original prototype.

To do this, we started by asking informants to tell us about a recent time they had reminisced, both to open the conversation and to engage them in thinking about how they reminisce. We then asked a number of questions about their reminiscence practices: what topics and periods of their life they reminisced about, how reminiscing made them feel, how they came to start and stop reminiscing, and so on. We then asked them about their practices around creating, preserving, and using mementos such as pictures, journals, and objects that can trigger reminiscence. Finally, we briefly described the initial prototype and asked them to react to it. We transcribed the interviews and members of the research team independently read them, looking for themes that emerged across multiple informants. Below we present the themes that had the strongest support.

Spontaneous and beneficial. Informants described reminiscing as mostly involuntary, triggered by external causes such as other people, sounds, and text materials. Aligning with earlier work [16], they described a variety of benefits of reminiscing, including connecting to people and learning from the past. They also tended to focus on positive rather than negative memories.

By, with, and about people. People are a central focus of reminiscing. All informants talked about other people triggering episodes of reminiscing. Most reported using reminiscing as a way both to get to know new people and to deepen connections with friends. Most also reported that compared to places or life eras, people are the dominant subject of reminiscing. "Well I'd say it was usually about people. And it's also partially about places but the places are important probably because of the people."

Privacy and intimacy. The meaning of reminiscing changes based on informants' relationship with the people they share memories with. "I think its very different with different people. With people you shared the experience with...it's a type of bonding but it's like, remembering why you're bonding. It's connecting. But when you're doing it with strangers or different people at the dinner table...it's more of a getting to know you." Further, the ways people reminisce by themselves and the topics they reminisce about are different than when they reminisce with others. "It's like things that we did, like that was a fun camping trip or something like that... When I'm by myself it's more personal experiences."

Making and use of mementos. Apart from other people, informants reported that photos were by far the most common memento they created and used in reminiscing. Journals were also common, while other physical mementoes, smells, songs, and newspaper clippings sometimes triggered reminiscence. Informants reported that in general, they wished they were more organized and consistent in how they cre- 
ated and organized their mementoes - and that the act of reminiscing sometimes made it harder to manage them. "I go through them intending to sort them, and then, I think of a bunch of memories, and then I don't get anything sorted."

Prompting seems promising. Informants liked the idea of being prompted to reminisce. They had trouble estimating how often they reminisced - a question worth following up with experience sampling - but wished they reminisced more. They saw this as a tool that could help.

Spontenaiety versus control. Although informants tended to experience reminiscence as spontaneous, most wanted control over when and how they received reminders, especially at work: "Serendipitous probably, but able to turn it off." People were lukewarm about text messages because they wanted to be able to control their level of interruption, and suggested email and IM as appropriate delivery tools. However, some informants were afraid that reminders too far in the background would be too easy to ignore.

Randomness and context. Informants saw value in randomly selected reminders, but also thought that choosing relevant prompts (e.g., created on this date, or related to recent people one has talked to or blog entries) might be valuable. "It would feel like an extension of myself in a way, like my subconscious that I'm not really aware of."

Using others' mementos. Informants also liked the idea of using reminders created by their friends, such as photos or status updates posted on Facebook. "My partner has all the VHS tapes of her kids when they were little and we've been watching them... even though I wasn't there, I wasn't involved, just being a part of it is like, 'wow, this is neat'. Like being in her past." Some also wanted to use prompts to reminisce not just about other people, but with them.

\subsection{Emailing impersonal prompts}

One of the key lessons from the interviews was that although many people have photos and journals, these are often not in a digital format. They also reported that items such as songs, generic pictures such as Christmas scenes or war pictures, smells, and newspaper items could trigger reminiscence through their personal associations with the triggering item, in agreement with prior work $[9,17]$.

This led us to explore whether impersonal prompts would help people remember to reminisce. Designing prompts was challenging; unlike in therapeutic groups where the facilitator can guide conversation, these prompts had to stand on their own. We wanted prompts to be appropriate to a wide audience, so we favored topics that many people could relate to and tried to avoid prompts that might lead to potentially embarrassing or offensive memories. We also tried to avoid prompts that turned reminiscing into a retrieval task that asked people to find a specific memory ("the best", "the most", "your favorite"), instead attempting to create more evocative prompts. We ended up with 95 prompts spanning topics ranging from family and friends to food and fun. Table 1 shows examples of prompts that generate many or few comments from users of the current prototype. ${ }^{2}$

\footnotetext{
${ }^{2}$ We have just begun to analyze the data collected by the
}

Table 1: Examples of impersonal prompts that have generated more or fewer comments from users of the current version of the prototype.

\begin{tabular}{|l|}
\hline Prompts that tended to generate many reactions. \\
\hline $\begin{array}{l}\text { The games you played most often with your siblings as } \\
\text { a child, perhaps something you did that would've gotten } \\
\text { you in trouble with your parents. }\end{array}$ \\
\hline $\begin{array}{l}\text { The song (or songs or album or albums) that defines } \\
\text { your teenage-hood. }\end{array}$ \\
\hline Your favorite weather and the last time you saw it. \\
\hline The way the first hot day in spring feels. \\
\hline $\begin{array}{l}\text { Your first job. How did you get it, and who were your } \\
\text { coworkers? }\end{array}$ \\
\hline The smell of someone you care for. \\
\hline Your favorite breakfast cereal. \\
\hline The first ethnic food that you ate. Did you like it? \\
\hline The last time you got a good night's sleep. \\
\hline $\begin{array}{l}\text { Play dates with friends as a child, perhaps your first or } \\
\text { a memorable sleepover. }\end{array}$ \\
\hline $\begin{array}{l}\text { Your commute to work. What were landmarks that you } \\
\text { were particularly fond of? }\end{array}$ \\
\hline $\begin{array}{l}\text { The best concert you went to. Who did you go see and } \\
\text { who did you go with? }\end{array}$ \\
\hline Candy bars you particularly enjoy. \\
\hline Some of the nicknames that you've had. \\
\hline $\begin{array}{l}\text { Your family's Thanksgiving traditions. What do you } \\
\text { eat? Is there a separate kids' table? }\end{array}$ \\
\hline $\begin{array}{l}\text { The nicest bathroom you've used. Where was it, and } \\
\text { why did you like it so much? }\end{array}$ \\
\hline
\end{tabular}

Prompts that generated relatively few reactions.

Meals that your mother made most often, or that you remember most as part of her repertoire. Did you have a particular meal of hers that you hated? That you loved? Your favorite room in the house you grew up in or your own bedroom.

The hallways in your school building. Did you ever get lost at school, or did you have a certain hallway where the class bell was painfully loud?

Something embarrassing that happened to you and how you recovered from it.

What winter feels like in your house.

Going to the dentist.

The last sporting event you attended.

An item of clothing or an outfit that you particularly adored as a child.

Your first experience in a different culture from your own.

The most fun project that you did elementary school.

Books that make you think about people in your life.

A technology related disaster.

Your first day of school.

Your favorite book as a child. Did you have your parents

read to you, or did you read on your own?

The biggest pet-peeve you have about your family.

Getting-ready-for-bed rituals or habits.

Your favorite fast food, cereal box, or cracker jack toy.

What you had for lunch at school or work. 
We deployed a version of Pensieve that e-mailed randomly selected prompts twice a day for two weeks to ten people associated with the lab, including three of the authors. We used e-mail rather than SMS because of interview informants' reactions and the cost of SMS in the U.S.

Again, people generally reacted positively. The prompts were often valuable for reminiscing, although the lead author disliked them compared to personal blog entries. People also said email helped strike a balance between serendipity and control, supporting spontaneous reminiscence but allowing people to easily defer the prompt if necessary. People sometimes received the same prompt several times. Rather than being annoyed, they reported that it sometimes led to deeper or different kinds of reminiscence. Further, people never reported being unhappy to have received a prompt, even in cases when a prompt led to a potentially unpleasant memory (for example, "Your father's favorite pastime" led one person to reply "Does drinking count?").

They also offered several suggestions. Sometimes prompts did not help people reminisce, and in these cases people wanted to be able to request a new prompt right away. Finally, some people wanted to create their own prompts, both to use and to share with other people using the prototypeagain, aligning with both our interviews and prior work [7].

\section{NEXT STEPS AND QUESTIONS}

Our data suggests that using technology to prompt reminiscence in this way has the potential to support people's reminiscing activities, as well as posing a number of interesting questions around the design of such technologies. We have released a new, publicly available prototype called Pensieve that incorporates our design insights and that points toward the questions we hope to answer. ${ }^{3}$ The name is drawn from an artifact in the Harry Potter books that allows people to see their own and others' memories of the past, and although the correspondence is not perfect - the artifact supports perfect capture and lacks spontenaiety - we chose the name to evoke the general idea of reminiscing.

Pensieve's architecture has three parts: a server, an email interface to deliver memory triggers, and a web interface to control Pensieve, record memories, and provide limited social support for reminiscence. The server supports flexible plugging in of new media types and data sources, allowing us to send memory triggers drawn from a broad range of user-created content services and media types. The original release supported Flickr, Picasa, and impersonal prompts; in early May we added support for Blogger, Twitter, and last.fm. Of the 72 users, 18 have signed up for at least one personalized service; we hope to continue to add services, including Facebook and common blogging platforms, that will allow more people to receive personalized content.

People create accounts using the web interface. An account page allows them to control how often they receive triggers

current prototype, so here we refrain from making claims about kinds of prompts that are more or less likely to support reminiscence. Analysis of these data are ongoing.

${ }^{3} \mathrm{http}: / /$ cornellhci.org/pensieve was released in February 2009 and has 72 users as of the end of May.

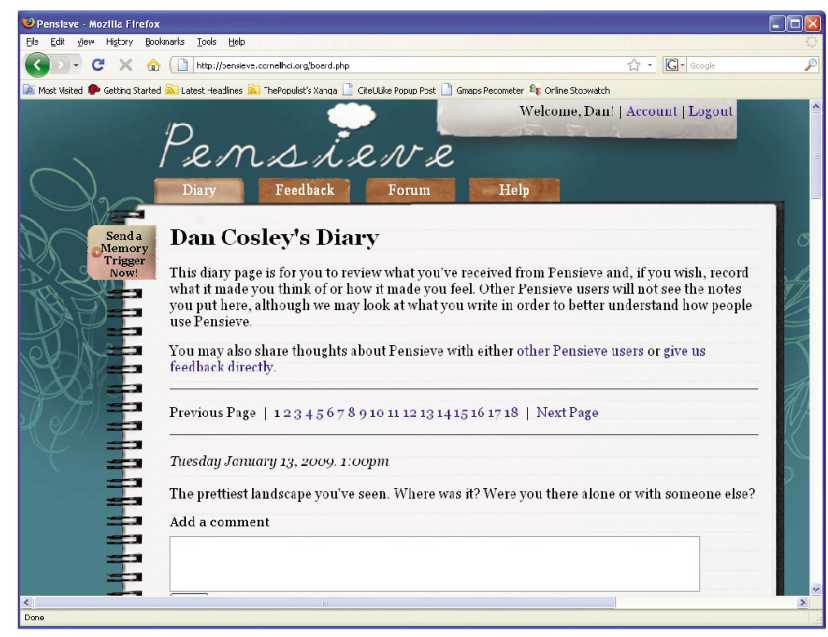

Figure 2: The diary page, where people can see and write about memory triggers they receive in email.

as well as which services they want to receive triggers from. People can sign up for multiple accounts per service, supporting both multiple personal identities and the ability to receive memory triggers from others in the way Twitter users can follow other users' tweets. After creating an account, most interaction happens through email. Pensieve randomly chooses a memory trigger either from one of a user's services or the impersonal prompts, then sends the trigger through email. The emails also contain links that encourage people to use features on the website.

After creating an account, the main aspect of the website is a diary page (Figure 2) that shows memory triggers Pensieve has sent. People can respond to triggers either in the diary or by replying to the email containing the trigger; these responses are shown in the diary. People have replied to about 800 of the 8,500 memory triggers Pensieve has sent, suggesting both that people do use the triggers to reminisce and that Pensieve may help people record their memories more regularly. For now, the diaries are private to prevent people from accidentally sharing sensitive memories. However, according with our interview subjects' reactions, some users wish for a more social experience.

The website does have a few social features. A forum page allows people to publicly discuss reminiscence or Pensieve itself. Finally, a feedback page allows people to submit impersonal prompts to to share with other people, answer provocative questions about Pensieve (loosely inspired by the ideas of cultural probes [4] and with the same goal of generating design insights), and provide general feedback. We hope this feedback, plus the ways people use the system, will allow us to answer a number of research questions.

What does it mean to effectively support reminiscence? The literature on reminiscence describes its use as a therapeutic tool [7], but metrics are hard to come by. We hope that we can combine indirect forms of feedback (entries on diary pages, submitted prompts) with occasional interviews to study how Pensieve affects, and hopefully supports, reminiscence. Several of our informants have reported thinking 
more about reminiscence and its role in their lives, just from having talked to us and used the tool.

Does this technology have legs? Although people's initial reactions have been almost uniformly positive, is this something that has long-term use and benefit for people? We plan to deploy Pensieve, in one form or another, for years, allowing us to collect longitudinal data.

Will people contribute content? We explicitly added the ability to write about the prompts one receives because our informants wanted to both reminisce and create mementos more often and more consistently. Will combining capture with reminiscing be useful? And will people really contribute to social goods such as the stock of impersonal prompts? We have some evidence for the former, but so far people have contributed relatively few prompts of their own.

How to integrate lifelogging technologies? Life logging systems might be quite useful if they can identify useful content and aspects of context that a reminiscing technology might use. "Lifestream" and other content aggregation technologies such as Joggle (http://beta.joggle.com/) might also be helpful. Finally, work on integrating digital and physical mementos [14] might inform future versions of the system.

How to use context? Although informants were happy with randomly selected memories, choosing relevant content might support better experiences. Simple but plausibly effective approaches might choose content created on this day $N$ years ago or, as one informant suggested, choose earlier content that is related to people's current activities.

Can we support social reminiscence? Privacy concerns led us to focus on individuals, but our informants' emphasis on people strongly suggests future designs that engage groups in reminiscing together. Pensieve has some social aspectsbeing able to access other users' social media streams, suggesting prompts for the community, and having a public forum for discussing reminiscence - and we are curious how those will be used. But we are really curious about how social technologies might change, and hopefully improve, people's reminiscing experiences. Could we use information on friendship ties provided by social networks when choosing which prompts to send, to whom, in order to stimulate conversations and reminiscing in groups?

These questions are important. Building and evaluating systems such as Pensieve highlights interesting social and computational challenges while potentially helping both individuals and groups reminisce more effectively. Tools like Pensieve may be especially useful for those with special needs, such as the elderly and Alzheimer's patients, and we hope to explore that in future work. But even simple technologies for collecting and reminding people to look at reminiscing content such as our prototypes, or the ubiquitous photo screensaver, seem to add a lot to people's lives. We hope our work here provides guideposts and stimulates other researchers' thinking on how to add even more.

\section{ACKNOWLEDGMENTS}

We would like to acknowledge Phil Adams for his contributions early in the project. This work was supported by NSF grant IIS 0845351 and Cornell University, as well as by Dr. Geri Gay.

\section{REFERENCES}

[1] T. Apted, J. Kay, and A. Quigley. Tabletop sharing of digital photographs for the elderly. In Proc. CHI '06, pages 781-790, 2006.

[2] F. Bryant, C. Smart, and S. King. Using the past to enhance the present: Boosting happiness through positive reminiscence. Journal of Happiness Studies, 6:227-260, September 2005.

[3] M. A. Conway and C. W. Pleydell-Pearce. The construction of autobiographical memories in the self-memory system. Psychol. Rev., 107(2):261-288, April 2000.

[4] B. Gaver, T. Dunne, and E. Pacenti. Design: Cultural probes. interactions, 6(1):21-29, 1999.

[5] J. Gemmell, G. Bell, and R. Lueder. MyLifeBits: a personal database for everything. CACM, 49(1):88-95, 2006.

[6] G. Hsieh, K. Wood, and A. Sellen. Peripheral display of digital handwritten notes. In Proc. CHI 2006, pages 285-288, 2006.

[7] H. F. Hsieh. Effect of reminiscence therapy on depression in older adults: a systematic review. International Journal of Nursing Studies, 40(4):335-345, May 2003.

[8] A. N. Joinson. Looking at, looking up or keeping up with people?: motives and use of facebook. In $\mathrm{CHI}$ '08, pages 1027-1036, 2008.

[9] M. Krakovsky. Nostalgia: Sweet remembrance. Psychology Today, May 2006.

[10] D. Petrelli, S. Whittaker, and J. Brockmeier. Autotopography: what can physical mementos tell us about digital memories? In Proc. $\mathrm{CHI}$ '08, pages 53-62, 2008.

[11] D. C. Rubin. Time in autobiographical memory. Cambridge University Press, 1996.

[12] A. J. Sellen, A. Fogg, M. Aitken, S. Hodges, C. Rother, and K. Wood. Do life-logging technologies support memory for the past?: an experimental study using sensecam. In Proc. CHI '0\%, pages 81-90, 2007.

[13] C. Shen, N. B. Lesh, F. Vernier, C. Forlines, and J. Frost. Sharing and building digital group histories. In Proc. CSCW '02, pages 324-333, 2002.

[14] M. M. Stevens, G. D. Abowd, K. N. Truong, and F. Vollmer. Getting into the Living Memory Box: Family archives \& holistic design. Personal Ubiquitous Comput., 7(3-4):210-216, 2003.

[15] J. D. Webster. Construction and validation of the Reminsicence Functions Scale. Journal of Gerontology, 48(5):256-262, September 1993.

[16] J. D. Webster and M. E. McCall. Reminiscence functions across adulthood: A replication and extension. J. Adult Dev., 6(1):73-85, January 1999.

[17] B. Woods, A. Spector, C. Jones, M. Orrell, and S. Davies. Reminiscence therapy for dementia. Cochrane Database of Systematic Reviews, 2005. 